\title{
Transcriptome analysis reveals unique C4-like photosynthesis and oil body formation in an arachidonic acid-rich microalga Myrmecia incisa Reisigl H4301
}

\author{
Long-Ling Ouyang, Si-Hong Chen, Yan Li and Zhi-Gang Zhou*
}

\begin{abstract}
Background: Arachidonic acid (ArA) is important for human health because it is one of the major components of mammalian brain membrane phospholipids. The interest in ArA inspired the search for a new sustainable source, and the green microalga Myrmecia incisa Reisigl H4301 has been found a potential ArA-producer due to a high content of intracellular ArA. To gain more molecular information about metabolism pathways, including the biosynthesis of ArA in the non-model microalga, a transcriptomic analysis was performed.

Results: The 454 pyrosequencing generated 371,740 high-quality reads, which were assembled into 51,908 unique sequences consisting of 22,749 contigs and 29,159 singletons. A total of 11,873 unique sequences were annotated through BLAST analysis, and 3,733 were assigned to Gene Ontology (GO) categories. Kyoto Encyclopedia of Genes and Genomes (KEGG) pathway analysis uncovered a C4-like photosynthesis pathway in M. incisa. The biosynthesis pathways of lipid particularly those of ArA and triacylglycerol (TAG) were analyzed in detail, and TAG was proposed to be accumulated in oil bodies in the cytosol with the help of caleosin or oil globule-associated proteins. In addition, the carotenoid biosynthesis pathways are discussed.

Conclusion: This transcriptomic analysis of $M$. incisa enabled a global understanding of mechanisms involved in photosynthesis, de novo biosynthesis of ArA, metabolism of carotenoids, and accumulation of TAG in M. incisa. These findings provided a molecular basis for the research and possibly economic exploitation of this ArA-rich microalga.
\end{abstract}

Keywords: Myrmecia incisa Reisigl H4301, 454 Pyrosequencing, C4-Like photosynthesis, Arachidonic acid, Microalga, Lipid metabolism, Oil body, Carotenoids

\section{Background}

Arachidonic acid (ArA, 20:4 $4^{\Delta 5,8,11,14}$ ), an $\omega$-6 long-chain polyunsaturated fatty acid (PUFA), is one of the major components of mammalian brain membrane phospholipids. It makes up approximately $20 \%$ of the total fatty

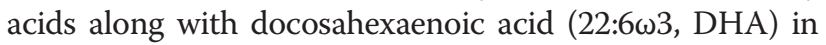
the brain [1]. In addition, ArA is an important precursor for the biosynthesis of diverse eicosanoids, such as prostaglandins, leukotrienes and thromboxanes, which play important roles in smooth muscle stimulation, platelet

\footnotetext{
* Correspondence: zgzhou@shou.edu.cn

College of Aqua-life Sciences and Technology, Shanghai Ocean University,

999 Hucheng Huan Road, Pudong New District, Shanghai 201306, China
}

aggregation control and the release of histamine during anaphylactic shock and other allergic reactions [2]. ArA deficiencies have been associated with neuro-visual development disorders and other premature birth complications [3-6]. ArA is therefore considered an essential nutrient in addition to DHA during early infant development and is suggested to be added into baby formula by the Food and Agriculture Organization (FAO). Besides, carotenoids are essential nutrients and important health beneficial compounds with respect to their antioxidant properties and ability to alleviate chronic diseases [7]. Human beings are mostly incapable to synthesize 
carotenoids de novo, therefore, they rely upon diet to obtain these compounds.

ArA can be commercially obtained from marine fish oil, animal tissues and fungi [8]. Interest in ArA and other long-chain PUFAs inspired the search for new PUFA sources. Myrmecia incisa Reisigl H4301, a coccoid green microalga species of Trebouxiophyceae [9], has recently been reported to accumulate an unprecedentedly high amount of ArA-rich triacylglycerols (TAG) in cytoplasmic lipid bodies [10]. When M. incisa was cultured under nitrogen starvation for $27 \mathrm{~d}$, its ArA content increased from $1.9 \%$ to $7.0 \%$ of dry weight (DW) biomass and $76 \%$ of the intracellular ArA accumulated in the form of neutral lipids [10]. In addition, carotenoids content increased as well under nitrogen starvation in Parietochloris incisa (synonym with M. incisa [11,12]) [13]. Thus, M. incisa may be a potential resource for both ArA and carotenoids exploitation.

There are two pathways proposed in microalgae for ArA biosynthesis [14-16]. The understanding of ArA biosynthesis pathway in $M$. incisa, thus, is first of all for the improvement of ArA level. Therefore, genes encoding fatty acid desaturases and elongase of this microalga have been characterized, and some routes were determined by heterologous expression of these genes in model organisms [10,17-19]. Carotenogenesis pathways in microalgae are similar to those in higher plants on one hand, and possess microalgae-specific features on the other hand [20]. Some common carotenogenesis genes have been identified in microalgae [20-22], whereas the molecular information for carotenoids biosynthesis in M. incisa is rather limited [10,11].

Expressed sequence tag (EST) analysis is the primary tool for novel gene discovery, particularly in non-model organisms for which full genome sequencing is not economically feasible. By using Sanger sequencing, a cDNA library from $M$. incisa generated only 595 unique sequences from 1,854 readable ESTs with little information of interest [11]. In contrast to this sequencing method, next-generation sequencing (NGS) technologies have led to a revolution in genomics and genetics and provided cheaper and faster delivery of sequencing information $[23,24]$. Currently, the newest 454 pyrosequencing platform, GS FLX Titanium, is one of the most widely used NGS technologies for the de novo sequencing and analysis of transcriptome in both the model organism of microalga, Chlamydomonas reinhardtii [25] and nonmodel ones including Alexandrium tamarense CCMP1598 [26], Botryococcus braunii BOT-88-2 [27], Botryococcus braunii BOT-22 [28], Dunaliella tertiolecta UTEX LB 999 [29] and Oxyrrhis marina [30].

In this paper, the 454 pyrosequencing method was used to gain more insight into the $M$. incisa metabolism pathways. Global transcriptome analysis revealed a C4- like photosynthesis pathway and a detailed lipid metabolism pathway. Furthermore, the identification of sequences coding for caleosin and oil globule-associated protein in the transcriptome of $M$. incisa provided evidence for accumulation of TAG in the form of oil body. Finally, the disclosure of the genes involved in the biosynthesis of carotenoids in $M$. incisa may favor the commercial exploitation of this algal resource in addition to ArA production.

\section{Results and discussion}

\section{4 pyrosequencing and de novo assembly}

In total, 381,804 reads (minimal size $>29$ bp) with an average length of 332 bp were generated from one pyrosequencing run. Filtering (e.g., removal of primers and poly A tail sequences) of the raw sequences resulted in 371,740 high-quality (HQ) reads with an average length of 321 nucleotides, generating $119 \mathrm{Mb}$ corresponding to $97.36 \%$ of the raw sequences (Table 1). After clustering and assembling using CAP3 clustering tools [31], these reads were assembled into 51,908 unique sequences consisting of 22,749 contiguous sequences (contigs) and 29,159 singletons (i.e., reads not assembled into contigs) (Table 1). Approximately $77 \%$ of the total contigs comprised 2 to 10 reads (Figure 1A). The average length of the total contigs, including $55.03 \%$ that ranged from 300 to $599 \mathrm{bp}$, was $642 \pm 438 \mathrm{bp}$, while the average length of singletons, including $89.25 \%$ that ranged from 100 to $499 \mathrm{bp}$, was $299 \pm 98 \mathrm{bp}$ (Figure 1B). In comparison with transcriptomes sequenced by 454 pyrosequencing from other microalgae, the HQ percentage of the $M$. incisa reads was nearly the same as that for D. tertiolecta but higher than that for Oxyrrhis marina (Table 2). In addition, the average length of the $M$. incisa reads was shorter than that of $D$. tertiolecta but longer than that of B. braunii BOT-88-2, B. braunii BOT-22 and Oxyrrhis marina (Table 2). This finding indicates that the sequencing data are creditable for further analysis. All of the HQ reads are available in the Sequence Read Archive (SRA) under the accession number SRA061977.

\section{Functional annotation}

A total of 7,361 contigs (32.36\% of total contigs) and 4,512 singletons (15.47\% of total singletons) had significant BLAST matches from selection of the best hits against Swiss-Prot, GenBank database and JGI Genome Portal at an e-value $\leq 10^{-3}$ (Table 1). The percent of annotated contigs was consistent with the $20 \%$ to $40 \%$ of the values previously reported for de novo eukaryotic transcriptome assemblies [32-34].

Analysis of the 11,873 BLAST-matched unique sequences suggested that there is less molecular information available for microalgae in comparison with other organisms in public databases as only approximately $42 \%$ of the 
Table 1 Summary of the transcriptome characteristics of $M$. incisa

\begin{tabular}{llll}
\hline & Total & Contigs & \\
\hline Number of raw reads & 381,804 & \\
Average nucleotide length of raw reads (bp) & 332 & 371,740 & \\
Number of HQ reads & 321 & 22,749 \\
Average nucleotide length of HQ reads (bp) & 51,908 & $642 \pm 438$ \\
Number of unique sequences & & 7,361 & 29,159 \\
Average nucleotide length of unique sequences (bp) & 11,873 & 2,199 & 4,512 \\
Unique sequences with BLAST matches & 3,733 & 2,019 & 1,534 \\
Unique sequences annotated with GO and KEGG pathways & 2,557 & 538 \\
Unique sequences assigned with EC numbers & & \\
\hline
\end{tabular}

BLAST-matched unique sequences were homologous to microalgae. However, unique sequences matched to genes from green microalgae accounted for 92\% approximately, demonstrating a distinct microalgae character for the transcriptome of $M$. incisa (Figure 2). The matched green microalgal species were mainly Chlamydomonas reinhardtii (Chlorophyceae), Volvox carteri (Chlorophyceae), Ostreococcus spp. (Mamiellophyceae) and Micromonas spp. (Mamiellophyceae) (Figure 2), of which Chlamydomonas reinhardtii is a model organism with a sequenced genome that has been the focus of most physiological, molecular and genetic studies [35]. The sequenced genomes of Ostreococcus tauri, Micromonas sp. RCC299 and Micromonas sp. CCMP1545 have offered valuable information for eukaryotic genome evolution and unusual photosynthesis apparatuses such as C4-like photosynthesis [36,37].

Of the 11,873 unique sequences with BLAST matches, $31.44 \%$ (2,199 contigs and 1,534 singletons) were annotated with GO terms, and $68.50 \%$ of the GO annotated unique sequences (2,019 contigs and 538 singletons) were assigned EC numbers (Table 1). One unique sequence may be assigned to several GO categories, and the distribution of the most abundant GO terms for molecular functions, biological processes and cellular components is presented in Figure 3. GO annotation for cellular component and biological process categories highlighted the dominance of unique sequences associated with metabolic processes in cell, especially in cytoplasm, with fewer unique sequences involved with cell differentiation $(0.5 \%)$, communication $(0.31 \%)$ and cellular component movement $(0.17 \%)$. The latter is consistent with the unicellular morphology without any flagella during the vegetative growth of $M$. incisa [11]. The molecular functional distribution indicated the top two assigned categories are binding processes (about 26\%) and catalytic activity (about 24\%). Besides, no pathogenesis process occurred in this microalga reflecting to some extent the axenic culture of $M$. incisa in this study.

However, this analysis only provided a general concept for the function of these unique sequences but no assignment for the metabolic pathways that they are involved in.
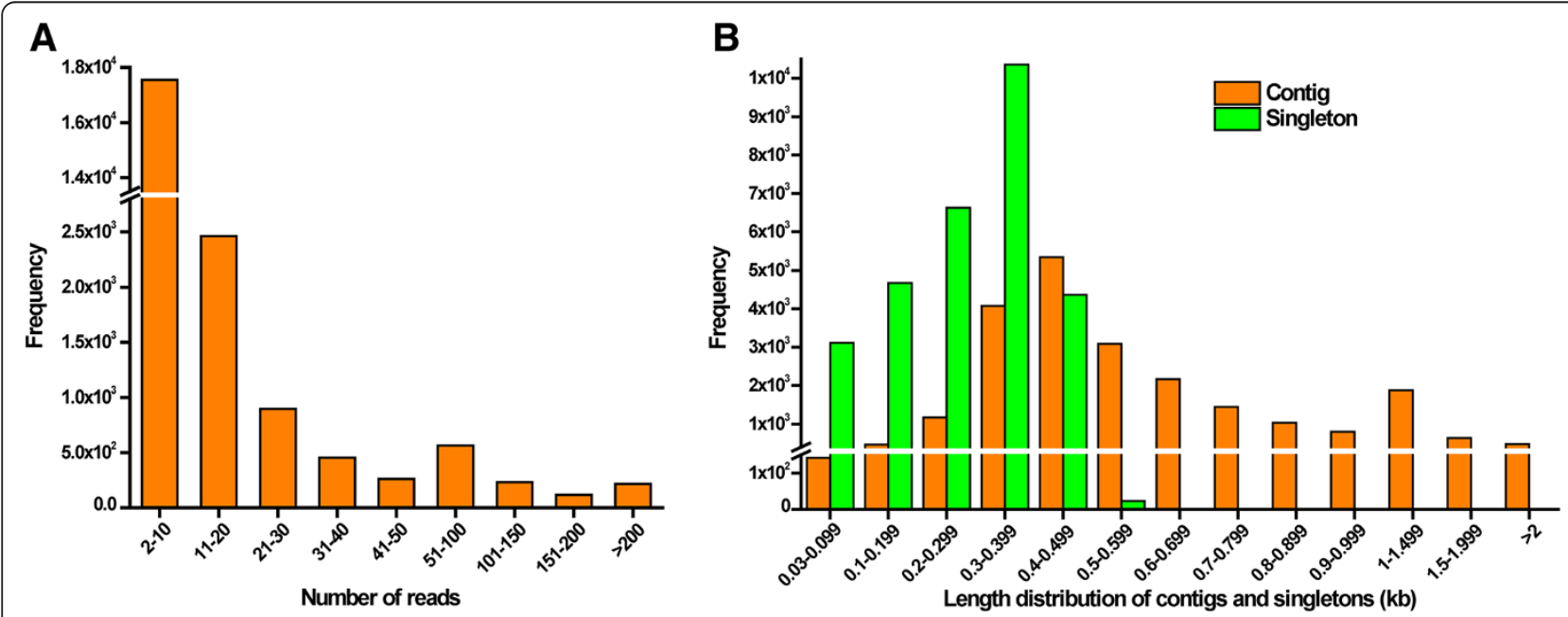

Figure 1 Overview of the assembly of the $\mathbf{M}$. incisa $\mathbf{H} 4301$ transcriptome. Distribution of number of reads assembled into contig (A) and the size distribution of the contigs and singletons (B). The unit of the $\mathrm{X}$-axis in Figure 1A is number of reads and in Figure 1B is kilobases (kb). 
Table 2454 pyrosequencing comparison between $M$. incisa and other microalgae

\begin{tabular}{|c|c|c|c|c|c|c|c|}
\hline Species & $\begin{array}{l}\text { Number of } \\
\text { raw reads }\end{array}$ & $\begin{array}{l}\text { Number of } \\
\text { HQ reads }\end{array}$ & $\begin{array}{l}\text { Percentage of } \\
\text { HQ reads }\end{array}$ & $\begin{array}{l}\text { Average length } \\
\text { of HQ reads (bp) }\end{array}$ & $\begin{array}{l}\text { Number of } \\
\text { contigs }\end{array}$ & $\begin{array}{l}\text { Number of unique } \\
\text { sequences }\end{array}$ & Reference \\
\hline Myrmecia incisa $\mathrm{H} 4301$ & 381,804 & 371,740 & 97.36 & 321 & 22,749 & 51,908 & This study \\
\hline Alexandrium tamarense & $N A^{a}$ & $1,073,382$ & NA & NA & NA & 40,029 & [26] \\
\hline Botryococcus braunii BOT-88-2 & NA & 185,936 & NA & 217 & NA & 29,038 & {$[27]$} \\
\hline Botryococcus braunii BOT-22 & NA & 209,429 & NA & 202 & NA & 27,427 & [28] \\
\hline Dunaliella tertiolecta UTEX LB 999 & $1,385,389$ & $1,365,353$ & 98.55 & 400 & 33,307 & 409,789 & [29] \\
\hline Oxyrrhis marina & 299,081 & 238,240 & 79.66 & 228 & 7,398 & 50,994 & {$[30]$} \\
\hline
\end{tabular}

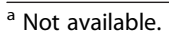

For further detailed understanding, unique sequences were then analyzed for $\mathrm{KO}$ identifiers using the KEGG database.

\section{Pathway assignment by KEGG}

Functional classification and pathway assignment was performed using KEGG with the annotated unique sequences. As shown in Additional file 1, the relatively complete pathways that the unique sequences were assigned to were related to translation, replication and repair, amino acid metabolism and energy and substance metabolism including glycolysis, the TCA cycle, pyruvate metabolism, photosynthesis, carbon fixation, starch and sucrose metabolism, arachidonic acid metabolism, glycerolipid metabolism and carotenoid biosynthesis. Particular attention focused on the biosynthesis pathways of ArA-rich TAG and carotenoids because $M$. incisa had the potential to accumulate these compounds, particularly under nitrogen starvation [10,13]. Interestingly, the assigned photosynthesis pathway suggested that $M$. incisa possessed some unique features for the carbon concentrating mechanism (CCM) as described below.
The C4-like photosynthesis pathway in $M$. incisa

The central carboxylation enzyme for $\mathrm{CO}_{2}$ fixation during photosynthesis is named ribulose-1, 5- bisphosphate carboxylase/oxygenase (RubisCO), but its affinity to $\mathrm{CO}_{2}$ is low [38]. To solve this problem, some plants, including several microalgae, such as Phaeodactylum tricornutum [39], Thalassiosira pseudonana [40], Ostreococcus tauri [36] and Micromonas sp. [37], have developed a C4-like photosynthesis pathway in addition to the biophysical CCM [bicarbonate transport and external or intercellular carbonic anhydrase (CA, EC 4.2.1.1)].

Based on the transcriptome analysis, it is interesting to find that $M$. incisa possibly possesses a C4-like photosynthesis pathway during the CCM. Several unique sequences encoding phosphoenolpyruvate carboxylase (PEPC, EC 4.1.1.31), phosphoenolpyruvate carboxykinase (PPCK, EC 4.1.1.32) and pyruvate orthophosphate dikinase (PPDK, EC 2.7.9.1) were identified (Additional file 2). Each of these enzymes is necessary for C4-metabolism. Three unique sequences encoding PEPC have been identified, including one that is 3,932 bp long with $71 \%$ amino acid identity with Coccomyxa subellipsoidea C-169 [GenBank: EIE27459]. In addition, the putative protein sequence of
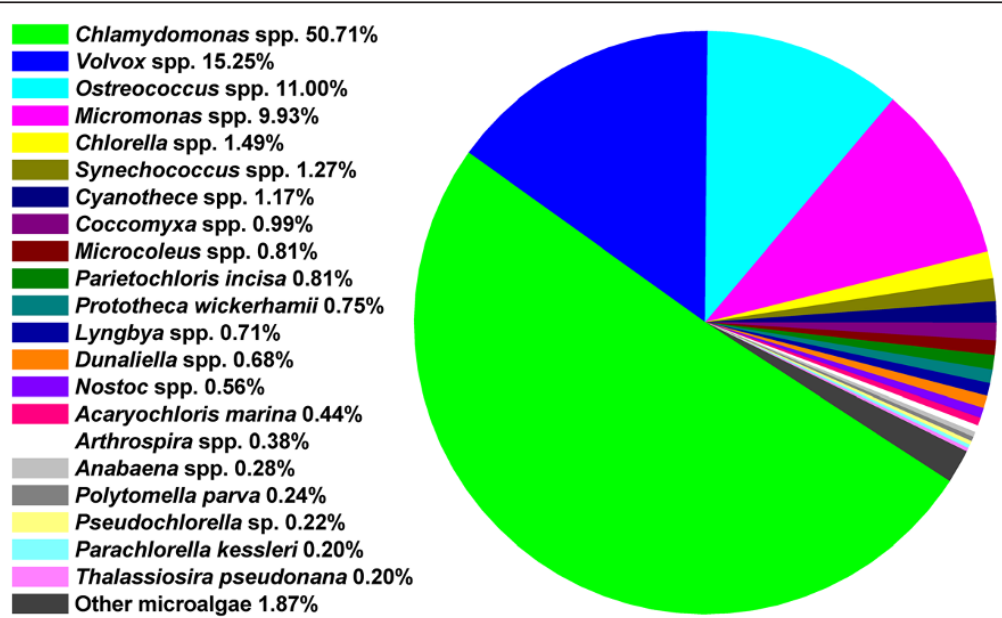

Figure 2 Distribution of microalgal species to which the unique sequences from $M$. incisa $\mathrm{H} 4301$ transcriptome were homologous by BLAST searches. 


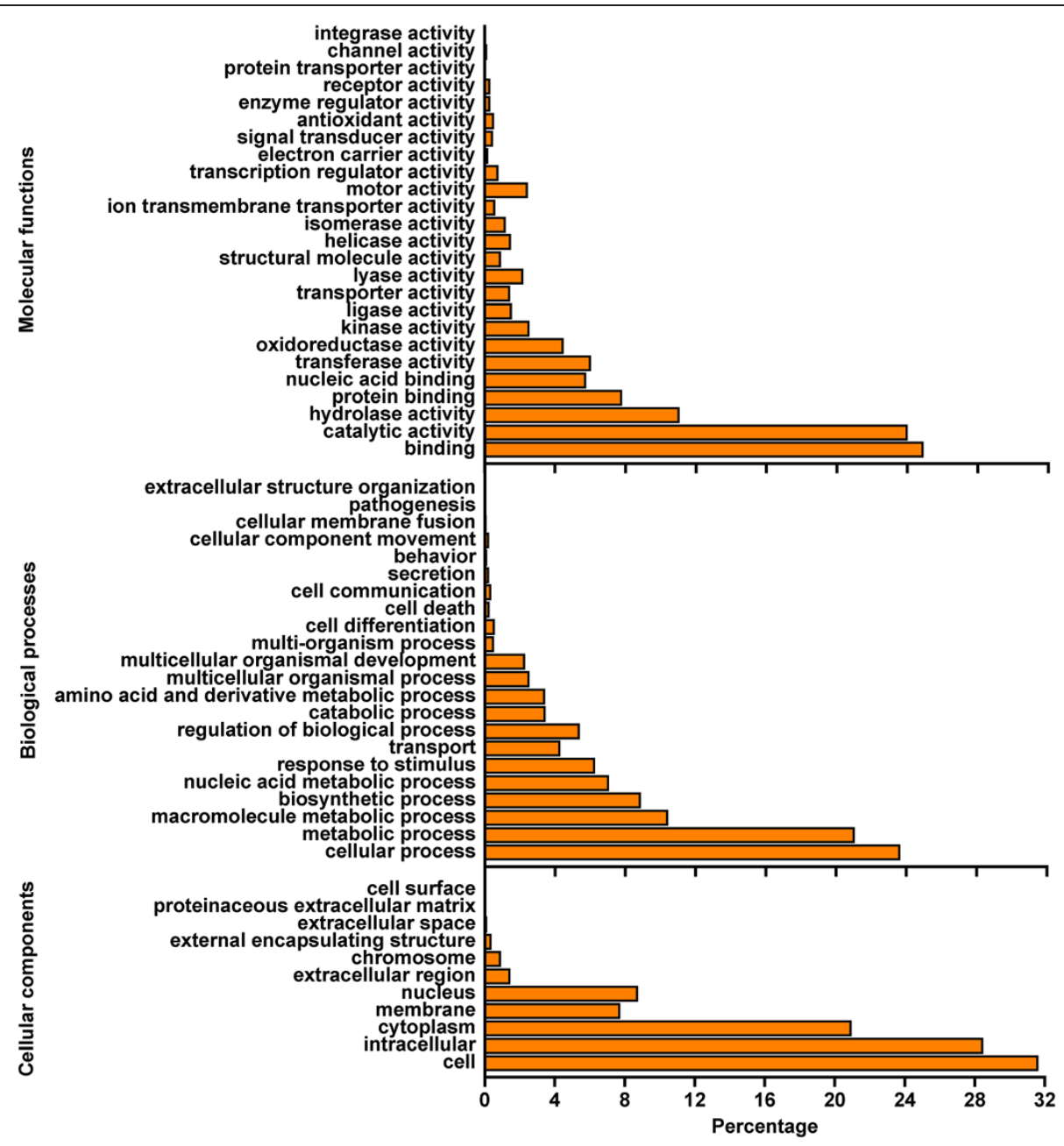

Figure 3 Gene ontology (GO) functional analysis of unique sequences from $M$. incisa $\mathbf{H} 4301$ transcriptome. Unique sequences were assigned to three categories: molecular functions, biological processes and cellular components.

this identified unique sequence revealed a PEP binding site, an $\mathrm{HCO}_{3}^{-}$trapping site and a C-terminal tetrapeptide QNTG [41,42]. Subcellular localization analysis predicted that this PEPC possesses neither a signal nor transit peptide (Additional file 2), suggesting that it may function within the cytosol to produce oxaloacetic acid (OAA).

On the one hand, OAA is assumed to be transaminated to aspartate (Asp) by Asp aminotransferase (AAT, EC 2.6.1.1) [43]. In fact, AAT can catalyze the reversible transfer of OAA and glutamate to Asp and $\alpha$-ketoglutarate. Eight unique sequences were identified to encode AAT, and one was cytoplasmic and another was mitochondrial (Additional file 2). The transaminated product in the cytosol, Asp, is subsequently imported into mitochondria via an Asp-glutamate carrier, which is important in malate/ Asp cycle [44]. One unique sequence in this transcriptome has been identified to have $53 \%$ identity with a mitochondrial isoform [GenBank: BAC84529] from Oryza sativa. Once Asp is transported into mitochondria, the mitochondrial AAT functions to regenerate OAA, which is then decarboxylated to PEP by a mitochondria-localized PPCK. Afterwards, the PEP may be converted to pyruvate with the concomitant phosphorylation of ADP to ATP by pyruvate kinase (PK, EC 2.7.1.40) catalysis. A NAD-

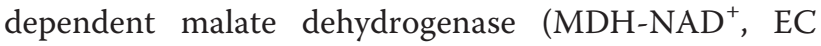
1.1.1.37) with a predicted mitochondrial transit peptide and its downstream enzyme NAD-dependent malic enzyme $\left(\mathrm{ME}-\mathrm{NAD}^{+}\right.$, EC 1.1.1.38), which has $46 \%$ identity with the mitochondrial ME-NAD ${ }^{+}$[GenBank: XP_002265765] from Vitis vinifera, were identified (Additional file 2). The resulting pyruvate from these two OAA decarboxylation pathways could participate in the TCA cycle.

On the other hand, OAA is assumed to be reduced to malate by NAPD-dependent MDH (MDH-NADP ${ }^{+}, \mathrm{EC}$ 1.1.1.82), which targets chloroplasts [45]. Although the mechanism of the OAA shuttle from the cytosol to chloroplast is unknown [39], a unique sequence encoding chloroplast-localized MDH-NADP ${ }^{+}$was identified in 
M. incisa. In addition, a putative NADP-dependent MEcoding unique sequence (ME-NADP ${ }^{+}$, EC 1.1.1.40) with $52 \%$ identity with a chloroplastic isoform [GenBank: XP_003546557] from Glycine max was annotated (Additional file 2). Dehydrogenated malate from OAA is decarboxylated by ME-NADP ${ }^{+}$to generate two different products $\mathrm{CO}_{2}$ and pyruvate. The former product is fixed by RubisCO along with the Calvin cycle, and the latter is converted by PPDK to PEP, which is then transported to the cytosol as the substrate of PEPC by PEP/Pi translocators. There are two putative chloroplast-localized $\mathrm{PEP} / \mathrm{Pi}$ translocators, and they have 74 and $52 \%$ identity with the chloroplastic isoforms from Brachypodium distachyon [GenBank: XP_003577954] and Coccomyxa subellipsoidea C-169 [GenBank: EIE22024] (Additional file 2), respectively. In addition, a unique sequence encoding a PEP/Pi antiporter was identified, demonstrating that there must be a PEP and Pi exchange between chloroplast and the cytosol in M. incisa (Figure 4). The predicted subcellular localization of PPDK, which had 96\% homology with that from Methylobacterium radiotolerans JCM 2831 [GenBank: YP_001755475], is unknown due to an incomplete sequence. However, the identified PEP/Pi translocator (antiporter) implied that there should be one chloroplastic PPDK in M. incisa.

In summary, the identification of the crucial enzymes of C4-like photosynthesis, such as PEPC, PPCK and PPDK, and the transporters highlighted the CCM in M. incisa. It possesses both CA (Additional file 2) and a C4-like photosynthesis pathway, thus enabling it to adapt to a low $\mathrm{CO}_{2}$ level habitat. It is another microalga in Chlorophyta with a C4-like CCM besides Ostreococcus tauri [36] and Micromonas sp. [37].

\section{De novo synthesis of fatty acids}

Global lipid biosynthesis pathways have been well known in many higher plants [46], whereas fatty acid biosynthesis and accumulation in microalgae have not been well studied because the genetic background of microalgae is limited. High-throughput sequencing, however, enabled us to understand lipid metabolism in B. braunii [27], D. tertiolecta [29] and M. incisa (Figure 5).

In all plants, palmitic (16:0) and oleic acid (18:1) are almost exclusively de novo synthesized in chloroplasts

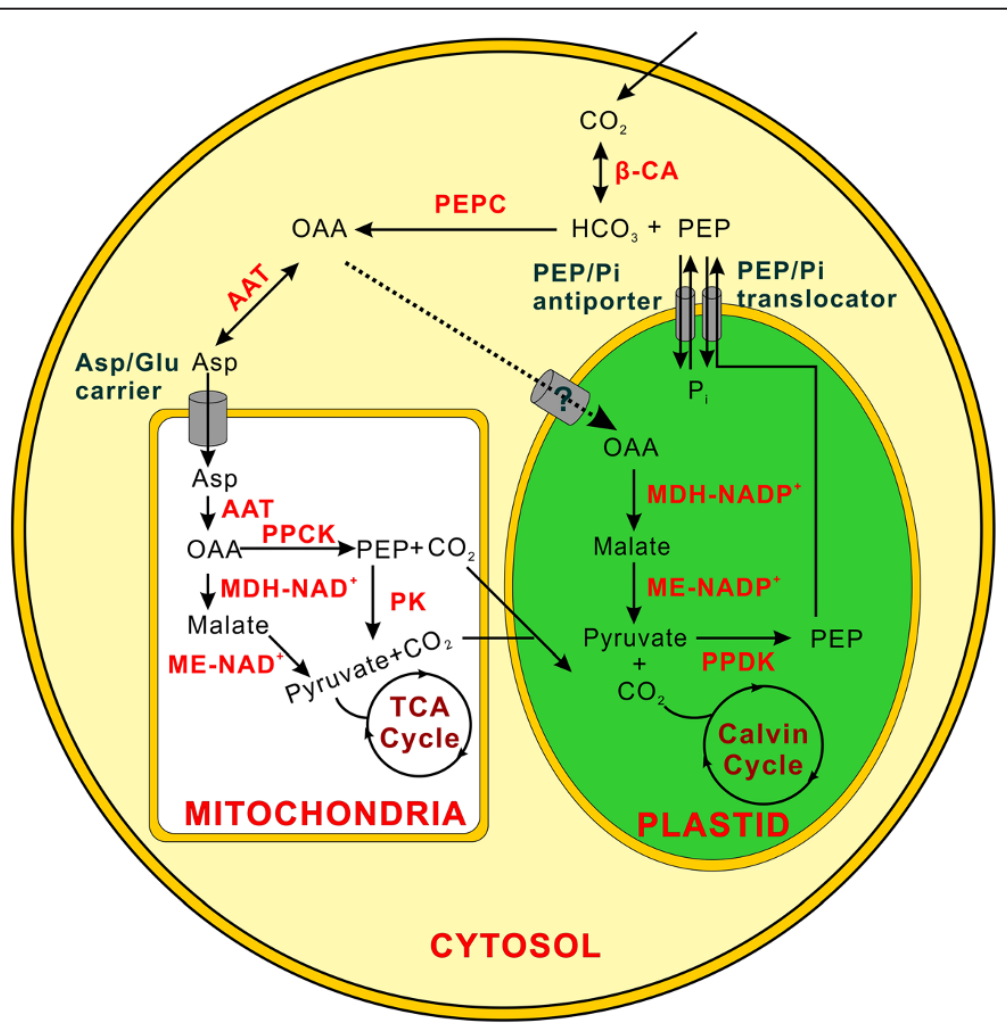

Figure 4 Model of the carbon concentration mechanisms in $\mathbf{M}$. incisa $\mathbf{H} 4301$ based on the transcriptome. The dashed line indicates the routes that was not represented in this transcriptome. Abbreviations are listed as follows: PEP, phosphoenolpyruvate; AAT, aspartate aminotransferase; OAA, oxaloacetic acid; Asp, aspartate; Pi, inorganic phosphate; CA, carbonic anhydrase; MDH-NADP ${ }^{+}$, NADP-dependant malate dehydrogenase; MDH-NAD ${ }^{+}$, NAD-dependant malate dehydrogenase; ME-NADP ${ }^{+}$, NADP-dependant malic enzyme; ME-NAD ${ }^{+}$, NAD-dependant malic enzyme; PEPC, phosphoenolpyruvate carboxylase; PPCK, phosphoenolpyruvate carboxykinase; PK, pyruvate kinase; PPDK, pyruvatephosphate dikinase; AAT, aspartate aminotransferase. 


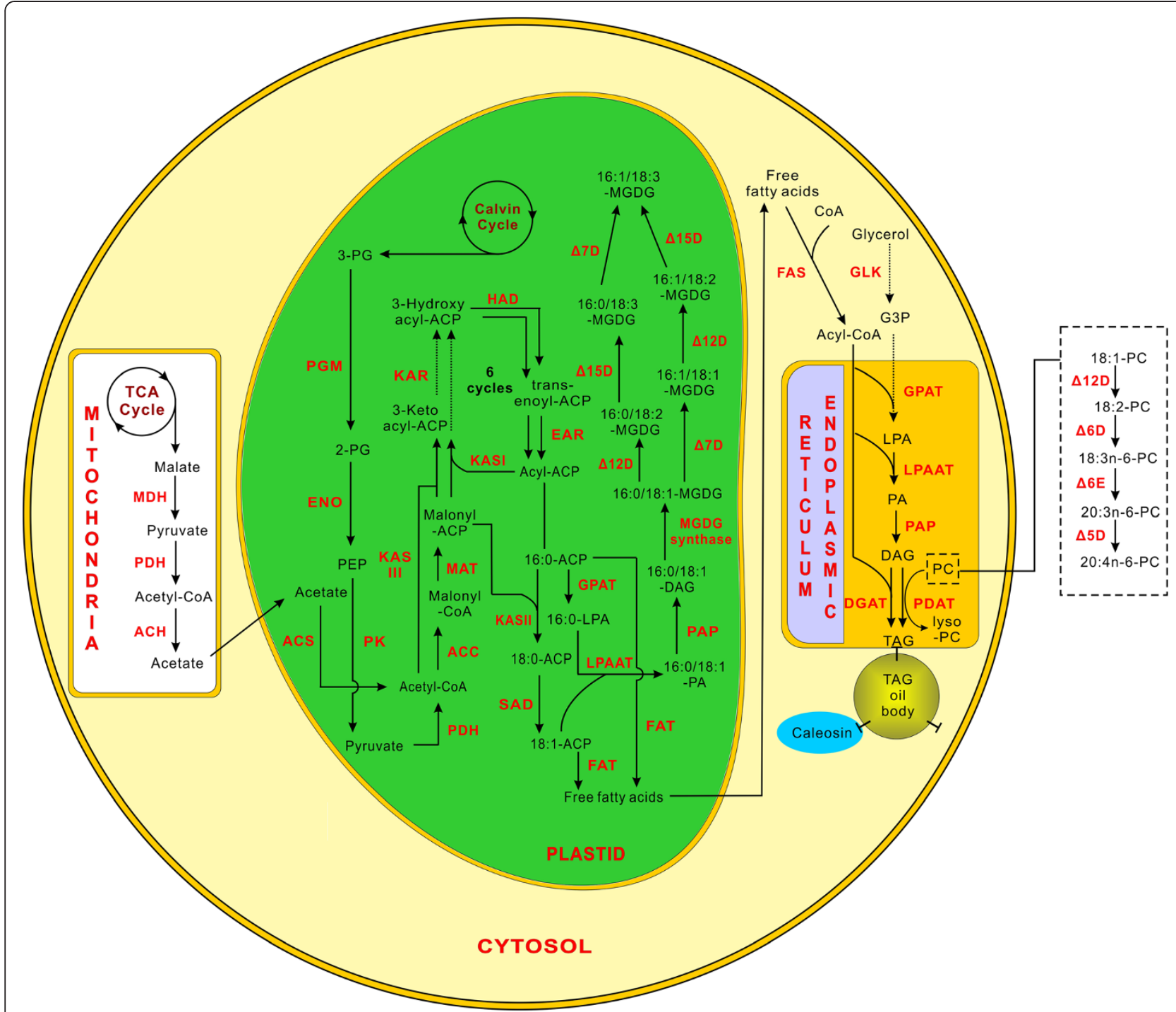

Figure 5 Fatty acid biosynthesis and their accumulation in $\mathbf{M}$. incisa $\mathbf{H} 4301$ based on the transcriptome. The dashed lines indicate the routes that were not represented in this transcriptome. Abbreviations are listed as follows: $M D H$, malate dehydrogenase; PDH, pyruvate dehydrogenase/decarboxylase; ACH, acetyl-CoA hydrolase; ACS, acetyl-CoA synthetase; KAS, 3-ketoacyl-ACP synthase; HAD, 3-hydroxyacyl-ACP dehydratase; KAR, 3-oxoacyl ACP reductase; MAT, malonyl-CoA:ACP transacylase; ACC, acetyl-CoA carboxylase; EAR, enoyl-ACP reductase; SAD, stearoyl-ACP desaturase; FAT, fatty acid thioesterase; PAP, phosphatidic acid phosphatase; MGDG, monogalactosyldiacylglycerol; $\Delta 9 D, \Delta 9$ desaturase; $\Delta 12 \mathrm{D}, \Delta 12$ desaturase; $\Delta 15 \mathrm{D}, \Delta 15$ desaturase; $\Delta 7 \mathrm{D}, \Delta 7$ desaturase; $\Delta 5 \mathrm{D}, \Delta 5$ desaturase; $\Delta 6 \mathrm{E}, \Delta 6$ elongase; FAS, fatty acid synthase; GLK, glycerol kinase; GPAT, glycerol 3-phosphate acyltransferase; LPAAT, 1-acyl-sn-glycerol-3-phosphate acyltransferase; DGAT, diacylglycerol acyltransferase; PDAT, phopholipid:diacylglycerol acyltransferase.

via the key precursor acetyl-coenzyme A (acetyl-CoA) [47]. There are two possible processes for supplying acetyl-CoA: one is directly from plastid pyruvate through the catalysis of pyruvate dehydrogenase/decarboxylase (PDH, EC 1.2.4.1), and the other is an indirect regeneration from the imported mitochondrial acetate through the catalysis of acetyl-CoA synthetase (ACS, EC 6.2.1.1) in plastid. The generation of acetate in mitochondria begins as pyruvate with acetyl-CoA as an intermediate and is sequentially catalyzed by mitochondrial $\mathrm{PDH}$ and acetyl-CoA hydrolase (ACH, EC 3.1.2.20) [47]. Based on transcriptome analysis, unique sequences encoding chloroplastic and mitochondrial PDH have been successfully identified (Figure 5). In addition, the putative mitochondrial $\mathrm{ACH}$ and chloroplastic ACS were identified (Additional file 2), demonstrating consistency with the description by Harwood [47].

The carbon chain condensation reaction begins with acetyl-CoA via the initial catalysis by acetyl-CoA carboxylase (ACC, EC 6.4.1.2). The generated malonyl-CoA is subsequently converted into malonyl-acyl carrier protein (malonyl-ACP) by malonyl-CoA:ACP transacylase (MAT, 
EC 2.3.1.39), and the product, malonyl-ACP, participates in whole fatty acid synthesis as an all-important source of $2 \mathrm{C}$ units. To complete the following series of condensation reactions, 3-ketoacyl-ACP synthase (KAS), 3-oxoacyl ACP reductase (KAR, EC 1.1.1.100), 3-hydroxyoctanoyl -ACP dehydratase (HAD, EC 4.2.1.59) and enoyl-ACP reductase (EAR, EC 1.3.1.9) are involved. During this course, three condensing enzymes, KASI, KASII and KASIII, play different roles [47]. It has been reported that the subunits of KASI and KASII dimers are encoded by two closely related plastid genes [48]. However, KASI extends the carbon chain length from $4 \mathrm{C}$ to $16 \mathrm{C}$ during six rounds of $2 \mathrm{C}$ elongation, whereas KASII elongates palmitate (16:0) to stearate (18:0). KASIII, which is distinct from KASI and KASII and is found in all plants [49], catalyzes the initial condensation reaction using acetyl-CoA rather than acetyl-ACP as a substrate for plant fatty acid synthesis (Figure 5). In $M$. incisa, at least one putative unique sequence of each type of KAS was identified, suggesting that carbon chain condensation reactions in this alga are the same as that in most plants.

After the formation of ACP-bound 18:0, the first double bond is introduced via the catalysis of stearoyl-ACP desaturase (SAD, EC 1.14.19.2). This enzyme, which is present in the plastids of higher plants and algae, is the only soluble $\Delta 9$ desaturase $[50,51]$. In this transcriptome, one SAD-coding unique sequence with $65 \%$ homology with plastid acyl-ACP desaturase from Coccomyxa subellipsoidea C-169 [GenBank: EIE22226] was identified. Products of fatty acid de novo biosynthesis, ACP-bound 16:0 and $18: 1^{\Delta 9}$ may subsequently constitute the biomembrane as a structural component or be exported into endoplasmic reticulum (ER) for the biosynthesis of ArA as a storage lipid (Figure 5).

\section{Biosynthesis of ArA and TAG}

In eukaryotic algae, most of the desaturation of fatty acids is catalyzed by acyl-lipid desaturases rather than by acylACP desaturase. In chloroplasts, glycolipids, which mostly include monogalactosyldiacylglycerol (MGDG), serve as substrates for chloroplastic $\Delta 12(\Delta 12 \mathrm{D}$, EC 1.14.19.6) and $\omega 3$ desaturases. In this study, several unique sequences encoding putative chloroplastic $\Delta 12 \mathrm{D}$ and $\omega 3$ desaturases, which the latter has been confirmed to be $\Delta 15$ desaturase ( $\Delta 15 \mathrm{D}, \mathrm{EC}$ 1.14.19.-) [18], were identified. In addition, a full-length cDNA sequence of MGDG-specific palmitate $\Delta 7$ desaturase ( $\Delta 7 \mathrm{D}, \mathrm{EC}$ 1.14.99.-), which is involved in desaturation leading to the formation of $16: 1^{\Delta 7}$, was also identified in this transcriptome. The biosynthesis of PUFAs taking place in the ER is similar to that in chloroplasts with the exception of those utilizing phospholipids, i.e., phosphatidylcholine (PC), as substrates (Figure 5). During the culture of $M$. incisa, a comparison of fatty acid content between complete and nitrogen-deficient medium suggested a possible biosynthesis pathway for ArA, which begins with $18: 1^{\Delta 9}$ with $18: 2^{\Delta 9,12}, 18: 3^{\Delta 6,9,12}$ and $20: 3^{\Delta 8,11,14}$ as successive intermediates. In this process, the ER-harbored $\triangle 12 \mathrm{D}$, $\Delta 6$ desaturase ( $\Delta 6 \mathrm{D}$, EC 1.14.19.3), $\Delta 6$ elongase $(\Delta 6 \mathrm{E}$, EC 6.21.3.-) and $\Delta 5$ desaturase ( $\Delta 5 \mathrm{D}, \mathrm{EC1.14.99.-)}$ were involved; the genes encoding these enzymes have been cloned as well [10]. The result of this study and the cloned genes again confirmed the presence of an ArA biosynthesis pathway in this microalga. Moreover, three other $\Delta 5 \mathrm{D}$-coding unique sequences were identified in this transcriptome (Additional file 2). Although the subcellular localization of the other $\Delta 5 \mathrm{D}$ was unpredictable due to incomplete sequences, these sequences provided further information for the investigation of ArA and other PUFA biosynthesis. The capacity of PUFAs may enhance the fluidity of the phospholipid membrane, which makes it possible for M. incisa to withstand chilling or cold stress [52].

A previous study has reported that most of the intracellular ArA was accumulated in the form of TAG in $M$. incisa, particularly during nitrogen starvation [10]. During the accumulation process, glycerol-3-phosphate (G-3-P) and acyl-CoA serve as primary substrates in the Kennedy pathway (Figure 5). One of the substrates, G-3-P, is generated by glycolysis or the catalysis of free glycerol by glycerol kinase (GLK, EC 2.7.1.30). However, sequence coding for GLK was not identified in this study. The other substrate, acyl-CoA, is generated via the esterification of free fatty acids exported from chloroplasts with CoA. These two substances are acylated by a series of acyltransferases including the initial glycerol 3-phosphate acyltransferase (GPAT, E.C: 2.3.1.15). This enzyme catalyzes the acylation of the sn-1 position of G-3-P to produce 1-acyl-sn-glycerol3-phosphate (LPA) [53]. The sn-2 position of LPA is acylated by 1-acyl-sn-glycerol-3-phosphate acyltransferase (LPAAT, EC 2.3.1.51) to generate phosphatidic acid (PA). Unique GPAT- and LPAAT-coding sequences were identified in the M. incisa transcriptome. Subcellular localization prediction analysis demonstrated that GPAT might target chloroplasts, whereas the localization of LPAAT was unknown due to the lack of a complete sequence (Additional file 2). PA is subsequently converted into either diacylglycerol (DAG) by phosphatidic acid phosphatase (PAP, EC 3.1.3.4) or cytidine diphosphate-diacylglycerol (CDP-DAG) by phosphatidate cytidylyltransferase (EC 2.7.7.41), and these two types of lipids alternately generate various phospholipids. The storage lipid, TAG, is either formed via an acyl-CoA-dependent reaction or an acyl-CoA-independent reaction, and both of them use DAG as an acyl-acceptor. The acyl-CoA-dependent reaction, which is catalyzed by diacylglycerol acyltransferase (DGAT, EC 2.3.1.20), uses fatty acid acyl-CoAs as acyl donors, whereas the acyl-CoAindependent reaction is mainly catalyzed by a phospholipid: diacylglycerol acyltransferase (PDAT, EC 2.3.1.158) uses PC as acyl donors instead. DGAT has two homologs, DGAT1 
and DGAT2, and their coding genes belong to the cholesterol acyltransferase gene family and the monoacylglycerol acyltransferase gene family, respectively. In $M$. incisa, there are five unique sequences encoding DGAT, three were identified as DGAT1, and the rest were identified as DGAT2 (Additional file 2). In algae, membrane lipids, including photosynthetic ones, rapidly degrade with the accumulation of cytosolic TAG-enriched lipid bodies when cells encounter stress [54]. If a PDAT orthologue was identified, particularly in chloroplasts, then it is conceivable that PC or even galactolipids may be utilized as acyl donors in TAG synthesis [54]. Three unique sequences coding for PDAT in this transcriptome were all partial sequences; thus, subcellular localization could not be predicted. However, this finding has already demonstrated that $M$. incisa is able to convert these membrane lipids into storage TAG.

$M$. incisa has been reported to be able to accumulate TAG in densely packed oil bodies [10]. Oil bodies in higher plant seeds are the most prominent and well studied [55-57]. They are spherical organelles consisting of neutral lipids enclosed by a semi-layer membrane of phospholipids coated with proteins (Figure 5). Thus far, only two proteins associated with oil bodies have been well described: oleosin and caleosin. Oleosins are mostly found in higher plants and are thought to be important for oil body stabilization in the cytosol, while caleosins are thought to be ubiquitous in higher plants and algae and play an important role particularly in oil body degradation during seed germination [58,59]. In Chlamydomonas reinhardtii, oleosin-like and caleosin-like genes were identified in the genome, whereas the relevant proteins were not identified by proteomic analysis [60]. Two unique sequences encoding caleosin with an EF-hand domain were identified, whereas none of the oleosin coding unique sequences were identified in this ArA-rich microalga (Additional file 2), thus implying this caleosin may play an important role in oil body formation. In addition, two unique sequences encoding oil globuleassociated proteins were identified (Additional file 2), which may also be related to oil body formation. However, the functional characteristics of these two genes coding for caleosin and oil globule-associated protein need further studies.

\section{TAG degradation}

Degradation of the storage lipid TAG plays an important role in the reconstruction of the cytoplasmic membranes of organisms grown under favorable conditions. The complete TAG degradation process includes a series of hydrolysis reactions via lipase [61]. This reaction begins with the release of fatty acids from the sn- 3 or sn- 1 positions of TAG to form DAG by triacylglycerol lipase (TAGL, EC 3.1.1.3). DAG is subsequently hydrolyzed into a fatty acid and 2-monoacylglycerol (2-MAG) via the catalysis of diacylglycerol lipase (DAGL, EC 3.1.1.-). Isomerization of the latter product to 1(3)-monoacyl-sn-glycerols occurs to some extent, and these may be completely degraded to glycerol and free fatty acids by monoacylglycerol lipase (MAGL, EC 3.1.1.23) (Additional file 3). The $\alpha$ - and $\beta$-type DAGL and TAGL-coding unique sequences were identified in this transcriptome (Additional file 2). It is worth mentioning that caleosin, as indicated above, may also play a role in TAG degradation in $M$. incisa according to previous reports about seed development [59].

In summary, all of the identified enzymes described above provide evidence for a better global understanding of the lipid metabolism process in M. incisa (Figure 5 and Additional file 3), though further study is needed to confirm their functional characteristics.

\section{Biosynthesis of carotenoids}

In the past two decades, great advancements have been made in studies of carotenoid metabolism in higher plants and algae [62]. The entire biosynthesis process can be divided into the following major steps: (1) isopentenyl diphosphate (IPP) synthesis; (2) enzymatic phytoene formation reactions; (3) the desaturation (dehydrogenation) of phytoene to lycopene; (4) cyclization and the formation of $\alpha$ - and $\beta$-carotenes; and (5) the synthesis of carotenes derivatives [20].

There are two suggested pathways for IPP synthesis: the acetate-mevalonate pathway beginning with acetate in cytosol and the phosphoglyceraldehyde-pyruvate pathway beginning with pyruvate in chloroplasts [20]. There are no identified unique sequences encoding mevalonate kinase (MVK, EC 2.7.1.36), phosphomevalonate kinase (PMVK, EC 2.7.4.2) or mevalonate pyrophosphate decarboxylase (MPD, EC 4.1.1.33), which are all required for the former pathway. In contrast, most of enzyme-coding genes involved in the latter pathway have been identified, and they encode 1-deoxyD-xylulose 5-phosphate synthase (DXS, EC 2.2.1.7), 1deoxy-D-xylulose 5-phosphate reductoisomerase (DXR, EC 1.1.1.267), 4-(cytidine 5'-diphospho)-2-C-methylD-erythritol kinase (CMK, EC 2.7.1.148), 2-C-methylD-erythritol 2,4-cyclodiphosphate synthase (MCS, EC 4.6.1.12) and 1-hydroxy-2-methyl-2-(E)-butenyl 4diphosphate synthase (HDS, EC 1.17.4.3). However, several unique sequences encoding the rest of the enzymes, including 2-C-methyl-D-erythritol 4-phosphate cytidyltransferase (CMS, EC 2.7.7.60) and 1-hydroxy2-methyl-2-(E)-butenyl 4-diphosphate reductase (HDR, EC 1.17.1.2) in this pathway, were unfortunately missed (Additional file 4). It appears that $M$. incisa may utilize glucose as the sole carbon source to synthesize carotenoids, which is the same as that in Chlorella and Scenedesmus [63]. 
Products generated from the first step include IPP and dimethylallyl diphosphate (DMAPP), which may be interconverted by the catalysis of IPP isomerase (IPPi, EC 5.3.3.2). The condensation of three IPP molecules and one DMAPP molecule (Additional file 4) is catalyzed by geranyl diphosphate synthase (GPPS, EC 2.5.1.1), farnesyl diphosphate synthase (FPPS, EC 2.5.1.10) and geranylgeranyl diphosphate synthase (GGPPS, EC 2.5.1.29) in a step-by-step manner to generate geranyl-geranyl diphosphate (GGPP). In addition to being the precursor for carotenoids, GGPP is also the precursor of several other groups of metabolites including chlorophylls, ubiquinones and tocopherols. Therefore, following the condensation of two molecules of GGPP by phytoene synthase (PSY, EC 2.5.1.32) to form phytoene is a committed step in carotenoid biosynthesis. Phytoene subsequently undergoes four steps to form lycopene by the sequential catalysis of phytoene desaturase (PDS, EC 1.3.99.29), 广-carotene

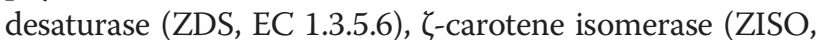
EC 5.2.1.12) and carotenoid isomerase (CRTISO, EC 5.2.1.13). In $M$. incisa, all of the enzymes except ZISO that are described in the lycopene formation process were successfully identified (Additional file 2).

Lycopene is an important intermediate in the biosynthesis of carotenoids, including $\beta$-carotene, which is responsible for yellow, orange or red pigments, photosynthesis and photo-protection in photosynthetic organisms. The cyclization of the lycopene catalyzed by lycopene $\varepsilon$ - and $\beta$-cyclases is a critical branch-point in carotenoid biosynthesis [64]. In one branch, a single enzyme, lycopene $\beta$-cyclase ( $\beta$-CYC, EC 5.5.1.19), introduces a $\beta$-ring at both ends of lycopene to form $\beta$ carotene in a sequential two-step reaction, while in the other branch, lycopene introduces one $\varepsilon$ - and one $\beta$-ring at each end via $\varepsilon$-CYC (EC 5.5.1.18) followed by $\beta$-CYC to form $\alpha$-carotene. Unique sequences encoding $\varepsilon$-CYC and $\beta-C Y C$ were identified (Additional file 2), suggesting that the biosynthesis pathway of $\beta$-carotene and $\alpha$-carotene is present in $M$. incisa. $\beta$-Carotene can be hydroxylated by $\beta$-carotene hydroxylase $(\mathrm{BCH}, \mathrm{EC}$ 1.14.13.129) in a two-step reaction to zeaxanthin with $\beta$-cryptoxanthin as an intermediate. In green tissues, zeaxanthin can be epoxidized to violaxanthin, and a set of light- and dark-controlled reactions known as the xanthophyll cycle rapidly optimizes the violaxanthin and zeaxanthin concentrations in the cell via the action of zeaxanthin epoxidase (ZEP, EC 1.14.13.90) and violaxanthin de-epoxidase (VDE, EC 1.10.99.3), respectively [65]. In this transcriptome, unique sequences that encode $\mathrm{BCH}, \mathrm{ZEP}$ and VDE were identified (Additional file 2). $M$. incisa is able to biosynthesize high-value carotenoids including, at the least, the $\alpha$ - and $\beta$-carotenes and zeaxanthin and violaxanthin. $\beta$-carotene is a precursor for vitamin A biosynthesis, which is important for growth and maintenance of the immune system and good vision $[66,67]$, and zeaxanthin and violaxanthin are usually used as food colourant. Due to this, further exploitation of this ArA-rich green microalga could be valuable.

\section{Conclusions}

In this study, 454 pyrosequencing provided a global understanding of the biosynthesis pathway in the non-model organism $M$. incisa. Based on the identified unique sequences and the subcellular localization of relevant proteins, a C4-like photosynthetic pathway was found to exist in $M$. incisa that enables this microalga to survive under low ambient $\mathrm{CO}_{2}[38,68]$. Nearly all unique sequences related to de novo ArA biosynthesis and TAG accumulation were successfully identified, thus demonstrating a more detailed lipid metabolism pathway. Furthermore, the identified unique sequences coding for PDAT, caleosin and oil globule-associated proteins helped to elucidate the mechanism of rapid accumulation and oil body formation of TAG under nitrogen starvation. In addition, the suggested carotenoid biosynthesis pathway enabled us to exploit this resource in addition to ArA production. All these unique sequences described above require functional identification prior to be used for genetically manipulating to enhance products of interest in further study.

\section{Methods}

\section{Algal species and culture}

The microalga Myrmecia incisa Reisigl H4301 was commercially provided by the Culture Collection Algae of Charles University of Prague (CAUP). A cell line isolated from this alga under a microscope was incubated in BG11 medium in 800-mL glass flasks, which were placed in a temperature-regulated photoincubator at $25^{\circ} \mathrm{C}$ and illuminated from the side with a light:dark regime of $12 \mathrm{~h}: 12 \mathrm{~h}$ using cool-white fluorescent Philips tubes (36 W) (Yizheng, Jiangsu, China) at a light irradiance of $115 \mu \mathrm{mol}$ photons $\mathrm{m}^{-2} \mathrm{~s}^{-1}$ [10]. During the culture, the flasks were shaken by hand several times every day at regular intervals. Algal cells were harvested with a centrifuge at 5,000 rpm in the late logarithmic growth phase and washed three times with nitrogen-free fresh BG-11 medium in which ferric ammonium citrate was substituted with ferric citrate. Subsequently, the algal cells were incubated under the mentioned conditions in fresh, nitrogen-free medium for an additional two days before harvest. The samples were washed with fresh medium without the addition of nitrogen, mixed together and stored in liquid nitrogen for total RNA extraction after centrifugation.

\section{RNA extraction and mRNA purification}

The frozen samples were ground with a pestle and mortar in liquid nitrogen, and the total RNA was then 
extracted using the TRIzol reagent (Invitrogen, USA) according to the manufacturer's protocol. Total RNA was dissolved in $200 \mu \mathrm{L}$ of RNase-free water. The total RNA concentration was determined by NanoDrop (Thermo Scientific, USA), and the RNA integrity value was evaluated using an RNA 6000 Pico LabChip Agilent 2100 Bioanalyzer (Agilent, USA).

Total RNA was incubated with 10 units of DNase I (Ambion, USA) at $37^{\circ} \mathrm{C}$ for $1 \mathrm{~h}$, and nuclease-free water was added to bring the sample volume to $250 \mu \mathrm{L}$. Messenger RNA was further purified with the MicroPoly (A) Purist Kit (Ambion, USA) following the manufacturer's protocol. The mRNA was dissolved in $100 \mu \mathrm{L}$ of RNA Storage Solution. The final concentration was determined using a NanoDrop.

\section{cDNA library construction and $\mathbf{4 5 4}$ pyrosequencing}

Double-stranded cDNA was synthesized from mRNA according to Ng's full-length cDNA synthesis protocol, with some modifications as described below [69]. A GsuI-oligo dT primer was used to prime first-strand cDNA synthesis from $10 \mu \mathrm{g}$ of mRNA using 1000 units of Superscript II reverse transcriptase (Invitrogen). After incubation at $42^{\circ} \mathrm{C}$ for $1 \mathrm{~h}$, the $5^{\prime}$-CAP structure of the mRNA was oxidized by $\mathrm{NaIO}_{4}$ (Sigma, USA) and ligated to biotin hydrazide (Sigma, USA), which was used to select complete mRNA/cDNA by binding with Dynal M280 beads (Invitrogen). After second-strand cDNA synthesis, the poly A and 5'-adaptor was deleted by GsuI digestion.

Complementary DNA size fractionation was performed using a cDNA size fractionation column (Agencourt, USA). Each cDNA fraction larger than 800 bp was sonicated to 300-800 bp and then pooled with the other cDNA samples ranging from $300 \mathrm{bp}$ to $800 \mathrm{bp}$. The prepared cDNAs were transformed into single-stranded template DNA (sstDNA) libraries using the GS DNA Library Preparation kit (Roche Applied Science).

Single-stranded template DNA libraries were clonally amplified in bead-immobilized form by using the GS emPCR kit (Roche Applied Science, USA) and sequenced on the 454 Genome Sequencer FLX System (Roche Diagnostic, USA).

\section{Bioinformatics analysis}

Using the SeqClean software (http://compbio.dfci.harvard. edu/tgi/software), low-quality (Q-value 20), low-complexity (poly A) and adaptor sequences were trimmed from the reads generated by the FLX sequencer and the qualified reads were then submitted to the CAP3 program [31] for clustering and assembly using default parameters. All unique sequences were deposited in the SRA of the National Center for Biotechnology Information (NCBI).

The open reading frame of each contig or singleton was identified using an in-house developed program based on 'GetORF' from EMBOSS with the parameters below: table $=0$, minsize $=90$ [70]. Gene annotation was performed using a BLASTp search against the Swiss-Prot and GenBank database with an E-value cutoff of $1 \times 10^{-3}$ and BLASTx against the JGI Genome Portal with an E-value cutoff of $1 \times 10^{-3}$ as well. The best gene annotation result was chosen by the two following principles: 1). the annotation with a clear functional description was firstly chosen; 2 ). the annotation with the best blast result (considering alignment coverage and identity) was chosen. Gene ontology (GO) analysis was performed using GoPipe [71] via BLASTP against the Swiss-Prot and TrEMBL databases with an E-value cutoff of $1 \times 10^{-3}$. The GI accessions of the best hits were retrieved, and the GO accessions were mapped to GO terms according to the molecular function, biological process and cellular component ontologies (http://www.geneontology.org/). A metabolic pathway was constructed based on the Kyoto Encyclopedia of Genes and Genomes (KEGG) database using the $\mathrm{BBH}$ (bi-directional best hit) method [72]. The analysis initially retrieved the KEGG orthology (KO) number for each protein, and metabolic pathways were then constructed based on the $\mathrm{KO}$ number. Targeting prediction was performed using TargetP 1.1 Server (http://genome.cbs.dtu.dk/services/TargetP/) and SignalP 4.1 Server (http://genome.cbs.dtu.dk/services/SignalP/).

\section{Additional files}

\section{Additional file 1: KEGG pathway annotation of the $M$. incisa H4301 transcriptome. \\ Additional file 2: Unique sequences involved in carbon concentrating mechanisms, lipid metabolism and carotenoid biosynthesis in $\boldsymbol{M}$. incisa $\mathbf{H 4 3 0 1}$. Abbreviations are listed as follows: CTP, chloroplast transit peptide; $\mathrm{MTP}$, mitochondrial targeting peptide score; $\mathrm{SP}$, signal peptide; $\mathrm{RC}$, reliability class ( $1=$ strong, $5=$ poor prediction) \\ Additional file 3: Triacylglycerol (TAG) degradation in $\mathbf{M}$. incisa H4301 based on transcriptome annotation. The unique sequence number of each identified gene is shown in parentheses. The dashed line indicates the route that was not represented in this transcriptome. Abbreviations are listed as follows: TAGL, triacylglycerol lipase; DAGL, diacylglycerol lipase; MAGL, monoacylglycerol lipase; 2-MAG, 2 monoacylglycerol; 1-MAG, 1-monoacylglycerol; FA, fatty acid.}

Additional file 4: Carotenoid biosynthesis model in M. incisa $\mathrm{H} 4301$ based on annotations in the transcriptome. Number of the identified enzyme-coding unique sequences is shown in the flow. The dashed lines indicate the routes that were not represented in this transcriptome.

Abbreviations are listed as follows: DXS, 1-deoxy-D-xylulose 5-phosphate synthase; DXR, 1- deoxy-D-xylulose 5-phosphate reductoisomerase; CMS 2-C-methyl-D-erythritol 4-phosphate cytidyl transferase; CMK, 4-(cytidine 5'-diphospho)-2-C-methyl-D-erythritol kinase; MCS, 2-C-methyl-D-erythritol 2,4-cyclodiphosphate synthase; HDS, 1-hydroxy-2-methyl-2-(E)-butenyl 4-diphosphate synthase; HDR, 1-hydroxy-2-methyl-2-(E)-butenyl 4diphosphate reductase; IPPi, isopentenyl diphosphate isomerase; GPPS, geranyl diphosphate synthase; FPPS, farnesyl diphosphate synthase; GGPPS, geranylgeranyl diphosphate synthase; PSY, phytoene synthase; PDS, phytoene desaturase; ZISO, zeta carotene isomerase; ZDS, zetacarotene desaturase; CRTISO, carotenoid isomerase; $\beta$-CYC, lycopene $\beta$ cyclase; $\varepsilon$-CYC, lycopene $\varepsilon$-cyclase; $\mathrm{BCH}$, $\beta$-Carotene hydroxylase; ZEP, 
zeaxanthin epoxidase; VDE, violaxanthin de-epoxidase; GADP glyceraldehyde 3-phosphate; DXP, 1-deoxy-D-xylulose 5-phosphate; MEP, 2-C-methyl-D-erythritol 4-phosphate; CDP-ME, 4-(cytidine 5'diphospho)-2-C-methyl-D-erythritol; CDP-MEP, 4-(cytidine 5'-diphospho)2-C-methyl-D-erythritol 2-phosphate; CMEPP, 2-C-methyl-D-erythritol 2,4-cyclodiphosphate; IPP, isopentenyl diphosphate; DMAPP dimethylallyl diphosphate; GPP, geranyl diphosphate; FPP, farnesyl diphosphate; GGPP, geranylgeranyl diphosphate.

\section{Abbreviations}

ArA: Arachidonic acid; PUFA: Polyunsaturated fatty acid; TAG: Triacylglycerol; DW: Dry weight; EST: Expressed sequence tag; NRS: Non-redundant sequence; HQ: High quality; GO: Gene ontology; KEGG: Kyoto encyclopedia of genes and genomes; KO: KEGG orthology; EC: Enzyme commission; RubisCO: Ribulose-1,5- bisphosphate carboxylase/oxygenase; CCM: Carbon concentrating mechanism; CA: Carbonic anhydrase; CoA: Coenzyme A.

\section{Competing interests}

The authors declare that they have no competing interests.

\section{Authors' contributions}

ZGZ participated in the design of the study. SHC carried out the experiments and acquisition of original data. LLO participated in sequence analysis and she and ZGZ were involved in data interpretation and drafting the manuscript. YL assisted with the bioinformatics analysis and helped to draft the manuscript, and ZGZ gave the final approval of the version to be published. All authors have read and approved the final manuscript.

\section{Acknowledgements}

This research was supported by the National Natural Science Foundation of China (31172389 and 30972243), the Special Project of Marine Renewable Energy from the State Oceanic Administration (SHME2011SW02), Shanghai Universities First-class Discipline Project of Marine Sciences and an Excellent Thesis Grant from Shanghai Ocean University.

\section{Received: 6 January 2013 Accepted: 6 June 2013}

Published: 13 June 2013

\section{References}

1. Crawford MA, Sinclair AJ: Nutritional influences in the evolution of mammalian brain. In Lipids, malnutrition and the developing brain. Edited by Elliott K, Knight J. London: Associated Scientific Publishers; 1972:267-292.

2. Lee JY, Hwang DH: Dietary fatty acids and eicosanoids. In Fatty Acids in Foods and Their Health Implications. 3rd edition. Edited by Chow CK. Boca Raton: CRC press; 2008:713-726.

3. Innis SM: Essential fatty acids in growth and development. Prog Lipid Res 1991, 30(1):39-103

4. Carlson SE, Werkman SH, Peeples JM, Cooke RJ, Tolley EA: Arachidonic acid status correlates with first year growth in preterm infants. Proc Natl Acad Sci U S A 1993, 90(3):1073-1077.

5. Crawford MA, Costeloe K, Ghebremeskel K, Phylactos A, Skirvin L, Stacey F: Are deficits of arachidonic and docosahexaenoic acids responsible for the neural and vascular complications of preterm babies? Am J Clin Nutr 1997, 66(Suppl 4):1032S-1041S

6. Lauritzen L, Hansen HS, Jørgensen MH, Michaelsen KF: The essentiality of long chain $\mathrm{n}-3$ fatty acids in relation to development and function of brain and retina. Prog Lipid Res 2001, 40(1-2):1-94.

7. Fraser PD, Bramley PM: The biosynthesis and nutritional uses of carotenoids. Prog Lipid Res 2004, 43(3):228-265.

8. Gill I, Valivety R: Polyunsaturated fatty acids, part 1: occurrence, biological activities and applications. Trends Biotechnol 1997, 15(10):401-409.

9. Reisigl H: Zur systematik und ökologie alpiner Bodenalgen. Österr Bot Z 1964, 111(4):402-499 (in German).

10. Ouyang L-L, Li H, Liu F, Tong M, Yu SY, Zhou Z-G: Accumulation of arachidonic acid in a green microalga, Myrmecia incisa $\mathrm{H} 4301$, enhanced by nitrogen starvation and its molecular regulation mechanisms. In Arachidonic Acid: Dietary Sources and General Functions. Edited by Dumancas GG, Murdianti BS, Lucas EA. New York: NOVA Science Publishers, Inc; 2013:1-20.

11. Ouyang L-L, Du DH, Yu SY, Li CY, Zhang CW, Gao HJ, Zhou Z-G: Expressed sequence tags (ESTs) analysis revealing the taxonomic position and fatty acid synthesis of an oleaginous green microalga, Myrmecia incisa Reisigl (Trebouxiophyceae, Chlorophyta). Chin Sci Bull 2012, 57(25):3342-3352.

12. Watanabe S, Hirabayashi S, Boussiba S, Cohen Z, Vonshak A, Richmond A Parietochloris incisa comb. nov. (Trebouxiophyceae, Chlorophyta). Phycol Res 1996, 44(2):107-108.

13. Merzlyak MN, Chivkunova OB, Gorelova OA, Reshetnikova IV, Solovchenko AE, Khozin-Goldberg I, Cohen Z: Effect of nitrogen starvation on optical properties, pigments and arachidonic acid content of the unicellular green alga Parietochloris incisa (Trebouxiophyceae, Chlorophyta). J Phycol 2007, 43(4):833-843.

14. Nichols BW, Appleby RS: The distribution of arachidonic acid in algae. Phytochemistry 1969, 8(10):1907-1915.

15. Shiran D, Khozin I, Heimer YM, Cohen Z: Biosynthesis of eicosapentaenoic acid in the microalga Porphyridium cruentum. I: The use of externally supplied fatty acids. Lipids 1996, 31(12):1277-1282.

16. Khozin I, Adlerstein D, Bigogno C, Heimer YM, Cohen Z: Elucidation of the biosynthesis of eicosapentaenoic acid in the microalga Porphyridium cruentum. Plant Physiol 1997, 114(1):223-230

17. Yu SY, Li H, Tong M, Ouyang L-L, Zhou Z-G: Identification of a $\Delta 6$ fatty acid elongase gene for arachidonic acid biosynthesis localized to the endoplasmic reticulum in the green microalga Myrmecia incisa Reisigl. Gene 2012, 493(2):219-227.

18. Li H, Ouyang L-L, Zhou Z-G: Low-temperature-induced expression of a $\omega 3$ fatty acid desaturase gene ( $\omega 3 F A D$ ) from Myrmecia incisa in Saccharomyces cerevisiae. J Agric Biotechnol 2012, 20(7):735-744 (in Chinese).

19. Liu F, Li H, Li CY, Ouyang L-L, Zhou Z-G: Characterization of several fatty acid desaturase (FAD) genes in Myrmecia incisa and their relative transcription affected by nitrogen starvation. J Fish Sci China 2012, 19(5):729-740 (in Chinese).

20. Takaichi S: Carotenoids in algae: distributions, biosyntheses and functions. Mar Drugs 2011, 9(6):1101-1118.

21. Bertrand M: Carotenoid biosynthesis in diatoms. Photosynth Res 2010 106(1-2):89-102

22. Capa-Robles W, Paniagua-Michel J, Soto JO: The biosynthesis and accumulation of beta-carotene in Dunaliella salina proceed via the glyceraldehyde 3-phosphate/pyruvate pathway. Nat Prod Res 2009, 23(11):1021-1028.

23. Mardis ER: The impact of next-generation sequencing technology on genetics. Trends Genet 2008, 24(3):133-141.

24. Morozova O, Marra MA: Applications of next-generation sequencing technologies in functional genomics. Genomics 2008, 92(5):255-264.

25. Miller R, Wu G, Deshpande RR, Vieler A, Gärtner K, Li X, Moellering ER, Zäuner S, Cornish AJ, Liu B, et al: Changes in transcript abundance in Chlamydomonas reinhardtii following nitrogen deprivation predict diversion of metabolism. Plant Physiol 2010, 154(4):1737-1752.

26. Moustafa A, Evans AN, Kulis DM, Hackett JD, Erdner DL, Anderson DM, Bhattacharya D: Transcriptome profiling of a toxic dinoflagellate reveals a gene-rich protist and a potential impact on gene expression due to bacterial presence. PLoS One 2010, 5(3):e9688.

27. Baba M, loki M, Nakajima N, Shiraiwa Y, Watanabe MM: Transcriptome analysis of an oil-rich race A strain of Botryococcus braunii (BOT-88-2) by de novo assembly of pyrosequencing CDNA reads. Bioresour Technol 2012, 109:282-286.

28. Ioki M, Baba M, Nakajima N, Shiraiwa Y, Watanabe MM: Transcriptome analysis of an oil-rich race $B$ strain of Botryococcus braunii (BOT-22) by de novo assembly of pyrosequencing CDNA reads. Bioresour Technol 2012, 109:292-296.

29. Rismani-Yazdi H, Haznedaroglu BZ, Bibby K, Peccia J: Transcriptome sequencing and annotation of the microalgae Dunaliella tertiolecta: pathway description and gene discovery for production of nextgeneration biofuels. BMC Genomics 2011, 12:148.

30. Lowe CD, Mello LV, Samatar N, Martin LE, Montagnes DJS, Watts PC: The transcriptome of the novel dinoflagellate Oxyrrhis marina (Alveolata: Dinophyceae): response to salinity examined by 454 sequencing. BMC Genomics 2011, 12:519.

31. Huang X, Madan A: CAP3: A DNA sequence assembly program. Genome Res 1999, 9(9):868-877.

32. Vera JC, Wheat CW, Fescemyer HW, Frilander MJ, Crawford DL, Hanski I, Marden JH: Rapid transcriptome characterization for a nonmodel organism using 454 pyrosequencing. Mol Ecol 2008, 17(7):1636-1647.

33. Meyer E, Aglyamova GV, Wang S, Buchanan-Carter J, Abrego D, Colbourne $\mathrm{JK}$, Willis BL, Matz MV: Sequencing and de novo analysis of a coral larval transcriptome using 454 GSFlx. BMC Genomics 2009, 10:219. 
34. Wang $H$, Zhang $H$, Wong $Y H$, Voolstra C, Ravasi T, Bajic VB, Quan P-Y: Rapid transcriptome and proteome profiling of a non-model marine invertebrate, Bugula neritina. Proteomics 2010, 10(16):2972-2981.

35. Merchant SS, Prochnik SE, Vallon O, Harris EH, Karpowicz SJ, Witman GB, Terry A, Salamov A, Fritz-Laylin LK, Maréchal-Drouard L, et al: The Chlamydomonas genome reveals the evolution of key animal and plant functions. Science 2007, 318(5848):245-250.

36. Derelle E, Ferraz C, Rombauts $S$, Rouzé $P$, Worden AZ, Robbens S, Partensky F, Degroeve S, Echeynié S, Cooke R, et al: Genome analysis of the smallest free-living eukaryote Ostreococcus tauri unveils many unique features. Proc Natl Acad Sci U S A 2006, 103(31):11647-11652.

37. Worden AZ, Lee J-H, Mock T, Rouzé P, Simmons MP, Aerts AL, Allen AE, Cuvelier ML, Derelle E, Everett MV, et al: Green evolution and dynamic adaptations revealed by genomes of the marine picoeukaryotes Micromonas. Science 2009, 324(5924):268-272.

38. Giordano $\mathrm{M}$, Beardall J, Raven $\mathrm{JA}: \mathrm{CO}_{2}$ concentrating mechanism in algae: mechanisms, environmental modulation, and evolution. Annu Rev Plant Biol 2005, 56:99-131

39. Kroth PG, Chiovitti A, Gruber A, Martin-Jezequel V, Mock T, Parker MS, Stanley MS, Kaplan A, Caron L, Weber T, et al: A model for carbohydrate metabolism in the diatom Phaeodactylum tricornutum deduced from comparative whole genome analysis. PLoS One 2008, 3(1):e1426.

40. Armbrust EV, Berges JA, Bowler C, Green BR, Martinez D, Putnam NH, Zhou S, Allen AE, Apt KE, Bechner $M$, et al: The genome of the diatom Thalassiosira pseudonana: ecology, evolution, and metabolism. Science 2004, 306(5693):79-86.

41. Izui K, Matsumura H, Furumoto T, Kai Y: Phosphoenolpyruvate carboxylase: a new era of structural biology. Annu Rev Plant Biol 2004, 55:69-84.

42. Kai $Y$, Matsumura H, Izui K: Phosphoenolpyruvate carboxylase: three dimensional structure and molecular mechanisms. Arch Biochem Biophys 2003, 414(2):170-179.

43. Andrews TJ, Johnson HS, Slack CR, Hatch MD: Malic enzyme and aminotransferases in relation to 3-phosphoglycerate formation in plants with the C4-dicarboxylic acid pathway of photosynthesis. Phytochemistry 1971, 10(9):2005-2013.

44. LaNoue KF, Schoolwerth AC: Metabolite transport in mitochondria. Annu Rev Biochem 1979, 48:871-922.

45. Slack CR, Hatch MD: Distribution of enzymes in mesophyll and parenchyma-sheath chloroplasts of maize leaves in relation to the C4-dicarboxylic acid pathway of photosynthesis. Biochem J 1969, 114(3):489-498.

46. Griffiths $G$, Stobart A: Fatty acid biosynthesis and triacylglycerol assembly. In Handbook of Food Enzymology. Edited by Whitaker JR, Voragen AGJ, Wong DWS. Boca Raton: CRC Press; 2002:125-145.

47. Harwood JL: Fatty acid biosynthesis. In Plant Lipids: Biology, Utilisation and Manipulation. Edited by Murphy DJ. Boca Raton: CRC Press; 2005:27-66.

48. Voelker T, Kinney AJ: Variations in the biosynthesis of seed-storage lipids. Annu Rev Plant Physiol Plant Mol Biol 2001, 52:355-361.

49. Walsh MC, Klopfenstein WE, Harwood JL: The short chain condensing enzyme has a widespread occurrence in the fatty acid synthetases from higher plants. Photochemistry 1990, 29(12):3797-3799.

50. Hildebrand DF, Yu K, McCracken C, Rao SS: Fatty acid manipulation. In Plant Lipids: Biology, Utilisation and Manipulation. Edited by Murphy DJ. Boca Raton: CRC Press; 2005:67-102.

51. Shanklin J, Somerville C: Stearoyl-acyl-carrier-protein desaturase from higher plants is structurally unrelated to the animal and fungal homologs. Proc Natl Acad Sci U S A 1991, 88(6):2510-2514.

52. Murata N, Sato N, Takahashi N, Hamazaki Y: Composition and positional distribution of fatty acid in phospholipids from leaves of chilling sensitive and chilling-resistant plants. Plant Cell Physiol 1982, 23(6):1071-1079.

53. Roughan PG, Slack CR: Cellular organization of glycerolipid metabolism. Annu Rev Plant Physiol Plant Mol Biol 1982, 33:97-132.

54. Hu Q, Sommerfeld M, Jarvis E, Ghirardi M, Posewitz M, Seibert M, Darzins A Microalgal triacylglycerols as feedstocks for biofuel production: perspectives and advances. Plant J 2008, 54(4):621-639.

55. Frandsen Gl, Mundy J, Tzen JTC: Oil bodies and their associated proteins, oleosin and caleosin. Physiol Plant 2003, 112(3):301-307.

56. Hsieh K, Huang AHC: Endoplasmic reticulum, oleosins, and oils in seeds and tapetum. Plant Physiol 2004, 136(3):3427-3434.

57. Huang AHC: Oil bodies and oleosins in seeds. Annu Rev Plant Physiol Mol Biol 1992, 43:177-200.
58. Murphy DJ: Lipid-associated proteins. In Plant Lipids: Biology, Utilisation and Manipulation. Edited by Murphy DJ. Boca Raton: CRC Press; 2005:226-258.

59. Weselake RJ: Storage lipids. In Plant Lipids: Biology, Utilisation and Manipulation. Edited by Murphy DJ. Boca Raton: CRC Press; 2005:162-225.

60. James GO, Hocart $\mathrm{CH}$, Hillier W, Chen H, Kordbacheh F, Price GD, Djordjevic MA: Fatty acid profiling of Chlamydomonas reinhardtii under nitrogen deprivation. Bioresour Technol 2011, 102(3):3343-3351.

61. Huang AHC: The biochemistry of plants. In Lipids: Structure and Function. Edited by Stumpf PK. Florida: Academic Press; 1987:91-119.

62. LU S, Li L: Carotenoid metabolism: biosynthesis, regulation, and beyond. J Integr Plant Biol 2008, 50(7):778-785.

63. Rohmer M: The discovery of a mevalonate-independent pathway for isoprenoid biosynthesis in bacteria, algae and higher plants. Nat Prod Rep 1999, 16(5):565-574.

64. Cazzonelli Cl, Pogson BJ: Source to sink: Regulation of carotenoid biosynthesis in plants. Trends Plant Sci 2010, 15(5):266-274.

65. Demmig-Adams B, Adams WW: Antioxidants in photosynthesis and human nutrition. Science 2002, 298(5601):2149-2153.

66. Van-Arnum SD: Vitamin A. In Kirk-Othmer Encyclopedia of Chemical Technology, Volume 25. 4th edition. New York: John Wiley; 1998.

67. Tanumihardjo SA: Vitamin A: biomarkers of nutrition for development. Am J Clin Nutr 2011, 94(2):658S-665S

68. Reinfelder JR: Carbon concentrating mechanisms in eukaryotic marine phytoplankton. Annu Rev Plant Biol 2011, 3:291-315.

69. Ng P, Wei CL, Sung WK, Chiu KP, Lipovich L, Ang CC, Gupta S, Shahab A, Ridwan A, Wong $\mathrm{CH}$, et al: Gene identification signature (GIS) analysis for transcriptome characterization and genome annotation. Nat Methods 2005, 2(2):105-111.

70. Rice P, Longden I, Bleasby A: EMBOSS: the European molecular biology open software suite. Trends Genet 2000, 16(6):276-277.

71. Chen Z, Xue C, Zhu S, Zhou F, Ling XB, Liu G, Chen L: GoPipe: streamlined gene ontology annotation for batch anonymous sequences with statistics. Prog Biochem Biophys 2005, 32(2):187-191.

72. Kanehisa M, Goto S, Furumichi M, Tanabe M, Hirakawa M: KEGG for representation and analysis of molecular networks involving diseases and drugs. Nucleic Acids Res 2010, 38(Suppl 1):D355-D360.

\section{doi:10.1186/1471-2164-14-396}

Cite this article as: Ouyang et al:: Transcriptome analysis reveals unique C4-like photosynthesis and oil body formation in an arachidonic acidrich microalga Myrmecia incisa Reisigl H4301. BMC Genomics 2013 14:396.

\section{Submit your next manuscript to BioMed Central and take full advantage of:}

- Convenient online submission

- Thorough peer review

- No space constraints or color figure charges

- Immediate publication on acceptance

- Inclusion in PubMed, CAS, Scopus and Google Scholar

- Research which is freely available for redistribution 